\section{MAKING THINGS BETTER? IMPLEMENTING THE LAST DAYS OF LIFE PERSONALISED CARE PLAN WITHIN AN ACUTE HOSPITAL TRUST}

Joanne Thorpe, Jayne Winter, Farida Malik, David Barclay. East Sussex Healthcare NHS Trust

10.1136/bmjspcare-2018-ASPabstracts.88

Local CQC inspection of one acute NHS Trust (2015), highlighted that improvement in recognising dying and individualised care planning for the dying person was required. In response, the Last Days of Life Personalised Care Plan (LDoLPCP) was developed based on NICE guidance and the five Priorities for care of the dying person (LACDP, 2014) and its use audited.

Methods A focussed support and education programme was used to develop and phase in the LDoLPCP. It has been used in all acute adult wards $(n=35)$ within the Trust since January 2017. From Jan-Jun 2017, an audit was carried out on a sample of notes of patients who had an expected death during an inpatient stay. Clinical notes were audited weekly using a proforma covering the five priorities for care of the dying person. The audit aimed to establish whether these patients had a LDoLPCP and if this was correctly completed. Data was collated anonymously and results analysed monthly and used in further education support.

Results There were 859 in-patient deaths, 267 patient notes included. In January 2017, 73.7\% (14/19) showed documented evidence of recognition that the patient may die within the next few hours/days, increasing to $84.9 \%(28 / 33)$ in June. Compliance in using the care plan increased from $57.9 \%(11 / 19)$ in January to $72.7 \%(24 / 33)$ in June. Utilising user feedback and audit results, version 2 of the LDoLPCP has been developed including an expanded emotional, spiritual and cultural needs section and how to explore these.

Conclusion The audit demonstrates the successful implementation of LDoLPCP with improvement in the number of people recognised as being in the dying phase having a personalised care plan. Weekly audits enabled concerns to be identified and education and support tailored to improve care plan use.

\section{MAPPING OF END OF LIFE RECOGNITION AND PALLIATIVE CARE PROVISION IN COPD}

Helen Ward, Frances Hakkak. The Royal Wolverhampton NHS Trust, Compton Hospice, Wolverhampton

\subsection{6/bmjspcare-2018-ASPabstracts.89}

Background COPD kills 25000 people per year in England and Wales. NICE guidelines suggest patients with end-stage COPD should have access to palliative services. GSF prognostic indicators assist clinicians to identify patients who are approaching end of life at an earlier stage enabling appropriate interventions to take place. These patients are more likely to receive well-coordinated and high-quality care. The aim of our study was to map the number of QoF registered COPD patients in Wolverhampton, the frequency of COPD specific GSF indicators, the number included on primary palliative care registers (\%o $¥ 2$ GSF prognostic indicators) and the number reviewed by palliative care.

Methods A multidisciplinary group with membership from CCG, acute trust, hospice and the community team oversaw the project. Data was collated from across all health care sites enabling us to confirm the number and type of GSF prognostic indicators for each patient and healthcare activity including palliative care reviews.

Results In March 2016 there were 4999 COPD patients; 52\% were male with an average age of 69.2 years. $24.6 \%$ of the patients $(n=1232)$ had \% $¥ 1$ GSF prognostic indicator. The most common indicator was MRC dyspnoea score of $4 / 5$ (58.4\%), followed by body mass index <20 (22.9\%). Of the 254 patients with \%o¥2 GSF prognostic indicators $15.4 \%$ were on the GP palliative care register. $20.1 \%$ of the 254 patients had been reviewed by the palliative care team.

Conclusion GSF prognostic indicators in COPD are prevalent with breathlessness being the most common. Only a small proportion of appropriate patients were included on the palliative care register (15.4\%) with more being seen by palliative care teams than on the registers. Further work is needed to ensure effective communication and education is provided across the whole health care system to identify patients earlier who are approaching end of life.

\section{ADVANCE CARE PLANNING IN HOSPITALISED FRAIL OLDER PATIENTS: A MISSED OPPORTUNITY? A SYSTEMATIC REVIEW AND NARRATIVE SYNTHESIS}

Sarah Hopkins, Allison Bentley, Veronica Phillips, Stephen Barclay. University of Cambridge

\subsection{6/bmjspcare-2018-ASPabstracts.90}

Background Frail older people have multiple hospital admissions in the last year of life and account for $40 \%$ of UK deaths. Advance care planning (ACP) has been shown to improve outcomes for patients and families. Most frail older people have not participated in ACP but would like to do so. We sought to understand whether an acute hospital admission represents an opportunity for frail older patients to engage in and benefit from ACP through a systematic review and narrative synthesis.

Method Electronic literature search of MEDLINE, CINAHL, ASSIA, PsycINFO, and Embase databases from 1 January 1990 to 1 September 2017. Population: Patients aged 60 years or older with multiple co-morbidities or frailty or need for help with personal care. Setting: Acute hospital wards in any healthcare system. Review questions: Does ACP occur and is it of benefit? What are the views of patients and clinicians? What are the barriers and facilitators?

Results The search returned 8076 unique articles, 16 of which met inclusion criteria. One further study was identified from reference searching. Rates of ACP with hospitalised frail older patients are very low $(0 \%-3 \%)$ but most patients would welcome the opportunity to participate in ACP, which was found to improve outcomes, including respecting patient wishes and reduced relatives' depression after death. Patients and families perceive the most important facilitator is access to doctors willing to discuss ACP, whereas clinicians believe the biggest barrier is family members' difficultly at accepting a poor prognosis. ACP uptake is increased by trained facilitators and patient ACP information packs.

Conclusions Hospital admission is a missed trigger opportunity for ACP with frail older patients, with a wide gap between evidence and clinical practice. Review findings are limited by variable study quality and scarcity of research in some areas. 